\title{
Visual prototype formation with discontinuous representation of dimensions of variability
}

\author{
PAUL G. NEUMANN \\ University of Miami, Coral Gables, Florida 39124
}

\begin{abstract}
The prototype-distance model (Posner, 1969) predicts that when a series of similar visual stimuli are experienced, a prototype is abstracted at the point in the multidimensional similarity structure which represents the greatest similarity to all stimuli, whether the elements of the prototype have actually been experienced or not. The attribute-frequency model (Neumann, 1974) predicts the prototype as a pattern composed of the most frequently experienced elements on each dimension of variability. In three experiments, it was determined that: (1) Under some conditions, a prototype is formed of unexperienced values, and, under other conditions, the best recognized stimuli are those incorporating the most frequent values; (2) the present form of the prototype-distance model cannot account for best recognized stimuli being other than the central tendency; and, (3) the attribute-frequency model can, in principle, account for either finding by incorporating additional assumptions about the specificity with which values on dimensions of variability are encoded.
\end{abstract}

One of the more viable theoretical models which attempts to account for the processes by which the cognitive system abstracts a "prototype" or "best example" from a set of similar patterns is that of Posner (1969). Posner has hypothesized that a set of similar patterns is best represented as points in multidimensional space. The multidimensional space incorporates one dimension for each dimension of similarity among the patterns. Each pattern may then be represented as the point at which the values incorporated on all dimensions of similarity intersect. The prototype is hypothesized to be that point in the multidimensional matrix which is the least distant from all other points: a kind of sophisticated multidimensional average. The membership value of each pattern in the set then becomes a function of its distance from the prototype in multidimensional space. The greater the distance which separates it from the prototype (as measured by some variant of the Minowski metric), the poorer a member it is.

Neumann (1974) has hypothesized that the structure of memory for such sets of similar patterns is best

This article is based on the empirical portion of a dissertation submitted in partial fulfillment of the requirements for the $\mathrm{PhD}$ degree at the University of Colorado. The research was supported by Grant BG $34077 \mathrm{X}$ to the Institute for the Study of Intellectual Behavior, Grant GJ 453 to the Computer Laboratory for Instruction in Psychological Research, both from the National Science Foundation, and by Grant MH 14314 to Lyle E. Bourne, Jr. from the National Institute of Mental Health. The author wishes to express his genuine gratitude to committee members Lyle E. Bourne, Jr. (chairman), William F. Battig, Peter G. Polson, Richard K. Olson, and Gary H. Stahl for professional guidance and personal encouragement well beyond the call of duty. Requests for reprints should be sent to Paul G. Neumann, Department of Psychology, Merrick 212N, University of Miami, Coral Gables, Florida 33124. represented in terms of the frequency distributions of experienced values across the dimensions of variability in the patterns. The prototype, or best example, is that pattern which incorporates the most frequently experienced value on each of the dimensions of variability. Other patterns derive their membership values as a function of the frequencies with which the values incorporated by each pattern are experienced. The best pattern is the one with the most frequent values; the worst one is the one which incorporates the least frequent values.

Ignoring, for the moment, that the mapping between dimensions of similarity and dimensions of variability may not be isomorphic (cf. Garner, 1974), a primary difference between the models lies in the measure of central tendency deemed appropriate: mean or mode. The derivation of the prototype in Posner's (1969) model lies in a multidimensional mean. In Neumann's (1974) attribute-frequency model, it is a multidimensional mode.

The distinction is not trivial. The prototype-distance model predicts that, under the proper circumstances, the prototype might be composed of values never experienced in the set of stimuli from which the prototype was abstracted, whereas the attribute-frequency model predicts that, although the prototype might be composed of combinations of values never experienced, the values themselves must be the most frequently experienced values on each dimension of variability. Exemplified in another fashion, the prototype-distance model predicts that, if the experienced values form a circle in a two-dimensional similarity structure, the prototype will be in the center of that circle, whereas the attribute-frequency model predicts that the best recognized stimulus must lie on the circumference. The analogy holds in an extension to a sphere in three dimensions. 
It should be noted that such an artifically constructed category, in which peripheral values are the most frequent, is likely to be at variance with the structure of many natural categories, given that most natural variables would seem to be normally distributed about some expectation. The attempt here is to construct a category of distinctive structure to determine if the structure which represents a category in memory is congruent with the experienced frequency distributions of attributes, or instead, if that structure, by nature of the cognitive processes operating on it, always represents the best example of a category as an "average." It is a question of how powerful are the assumptions which underly the processing. Do they "assume," in some sense, that all categories are structured such that exemplars are distributed as deviations from some expectation, even if the expectation is represented by values never experienced, or do the processes actually monitor the distributions and represent the category in a fashion congruent with experienced distributions of values?

The following experiments were designed to determine if, and under what circumstances, human subjects derive a prototype composed of unexperienced values on dimensions of variability in a set of similar visual stimuli.

\section{EXPERIMENT 1}

\section{Method}

Subjects. Subjects were nine undergraduate introductory psychology students at the University of Colorado, who volunteered for participation in partial fulfillment of optional course requirements.

Materials. A population of 125 faces, each duplicated, was constructed using an Identikit. The Identikit can be conceptualized as the modern replacement for the "police artist." It consists of many essentially clear plastic templates, each bearing some variation of a facial feature. For example, one set of templates bears many variations of hairstyles, another set bears many variations of eyebrows, another eye shapes, another noses, another lips, and so forth. A complete face may be constructed by overlaying a series of templates, using one for each feature. A single set of features was selected for the basic face. Since the templates bear information as to the identity of the template and alignment marks, reproductions of the templates were made, on which all information except the facial feature itself and peripheral alignment marks were removed by a series of technical photographic procedures. The faces produced with the templates varied on three dimensions, with five values on each dimension. The first dimension was age, accomplished by overlaying the basic face with either no age template or one of four templates bearing age lines, producing faces which varied from approximately 20 years of age to approximately 60 years of age in 10-year increments. The second dimension was the length of the face, variations in which were accomplished by raising the hairstyle template and lowering the chin-ears template in increments scaled on the edges of the templates. There are, appropriately, five such increments, producing five values of the length of the face. The third dimension was the length of the nose, accomplished by raising and lowering the nose template through its five scaled increments. Since the quality of faces produced in this manner is much like that of line drawings, the faces were photographed on high-contrast copy film, using a slightly reduced field of view to eliminate the peripheral scale marks. Positive transparencies were then produced by contact printing on high-contrast lithographic film. The result was a clear transparency with dark black reproduction of the facial features. The transparencies were then mounted in standard $51 \times 51 \mathrm{~mm}$ slide mounts for projection.

An acquisition set of faces was selected randomly, with the restriction that across the eight faces in the set, Values 1 and 5 on each dimension appeared three times each, Values 2 and 4 appeared once, and Value 3 did not appear, creating a $V$-shaped distribution across each dimension of variability and placing a void at the point which the prototype-distance model predicts as the locus of the prototype. A set of 15 recognition faces was assembled in much the same fashion, with the exception that the first block of five faces consisted of correlated-value faces $(1,1,1 / 2,2,2 / 3,3,3 / 4,4,4 / 5,5,5)$ yielding a crosssection of the three-dimension structure. The other 10 faces were randomly selected, with the restriction that each value of each dimension was represented once in each of the two blocks of five faces. The order of the faces was randomly generated within blocks of five.

Score sheets were generated by a simple FORTRAN IV program, which printed sets of five criterial sentences. The sentences read, in order: "I am sure that I did not see it," "I do not think that I saw it," "I do not know whether I saw it or not," "I think that I saw it," and "I am sure that I saw it." Thus, each time the subject evaluated a face in the recognition set, the sentences provided reinstruction as to the criteria for a 5 -point scale.

Procedure. Subjects were run in groups ranging from two to five, depending upon the number which had volunteered for the various sessions. They were seated at tables facing a dull white wall, upon which the faces were projected. They were informed that they would see a series of faces projected in front of them, that they would have $10 \mathrm{sec}$ to view each face, and that they were to try to remember exactly the faces they were shown.

Following projection of the acquisition set, subjects were told to remain silent for $10 \mathrm{~min}$, during which they should try to remember exactly which faces they had just seen. During the final $3 \mathrm{~min}$ of this period, the experimenter handed out the answer sheets, explained that they would see another series of faces which would remain visible for $15 \mathrm{sec}$ each, and that during this period they were to decide which of the five sentences most closely resembled their decision for the face and mark that sentence as their answer. After insuring that all subjects understood the task, the recognition set was projected. Following completion of the test trials, the experimenter collected the answer sheets.

Each protocol was scored in the following manner: The sentences appearing on the score sheet were assigned a numerical value ranging from 1 ("I am sure that $I$ did not see it") to 5 ("I am sure that I saw it"). The integer 3 is thus the dividing line between recognition and nonrecognition.

\section{Results}

Regression analyses were performed using distance from prototype and summed frequency of representation of each incorporated value in the pattern. The regression coefficients were negative, indicating that recognition decreased with increasing distance from the prototype, as predicted by the prototype-distance model, and decreased as the frequency sums increased, contrary to the predictions of the attribute-frequency model. Table 1 indicates that the regression on distance from the prototype is statistically significant.

The face which received the highest mean confidence 
Table 1

Regression Analyses for Experiment 1

\begin{tabular}{lcccrr}
$\begin{array}{c}\text { Independent } \\
\text { Variable }\end{array}$ & $\begin{array}{c}\text { Regression } \\
\text { Coefficient }\end{array}$ & $\begin{array}{c}\text { Regression } \\
\text { Mean Square }\end{array}$ & $\begin{array}{c}\text { Error } \\
\text { Mean Square }\end{array}$ & df & F \\
\hline Distance & -.309 & 5.7053 & .4911 & 1,13 & .04 \\
Frequency Sums & -.200 & 6.1920 & .4536 & 1,13 & 1.6177 \\
\hline
\end{tabular}

rating was the face composed of Value 3 on all dimensions of variability, as predicted by the prototypedistance model. Figure 1 illustrates the mean confidence ratings across the five correlated-value test faces. The distribution of mean confidence ratings across this cross-section of the three-dimensional variability structure clearly reflects the predictions of the prototype-distance model.

\section{Discussion}

The results of Experiment 1 clearly favor the predictions of a prototype-distance model over those of an attribute-frequency model. The face which received the highest mean confidence in recognition was a face composed of the central values $(3,3,3)$ on all dimensions of variability, none of which had been incorporated in any of the faces seen during the acquisition phase of the experiment. Faces composed of the most frequently experienced values, which were the extreme values of each dimension, received lower mean ratings than the face composed totally of unexperienced values.

In addition to the predictions of the prototypedistance model, however, there are at least two additional ways of accounting for the results. The stimuli were in the form of faces, with which subjects have had a large amount of previous experience. It would thus seem possible that recognition of a face of average proportions might be with reference to a previously acquired expectation on the dimensions of variability, and that the brief exposure in the laboratory to a distribution of facial attributes in which

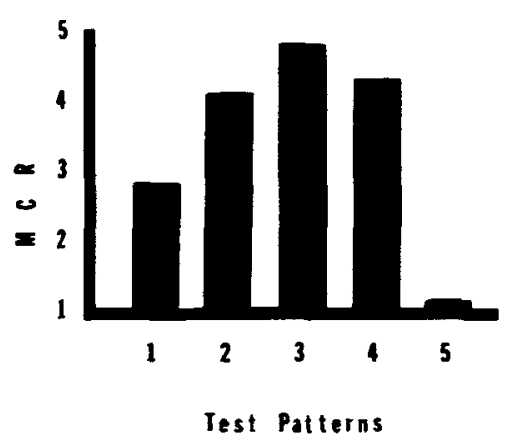

Figure 1. Mean confidence ratings for the five correlatedvalue test patterns in Experiment 1. The number of the pattern corresponds to the value represented on all dimensions. A mean of 3.0 is the dividing point between recognition and nonrecognition. the extremes are more frequent than the central values may not be sufficient to override, even temporarily, the overwhelming effects of long experience with facial attributes.

Another possibility lies in the difference between simultaneous and successive discriminations. Although comparison of two faces reveals a clearly discriminable difference, the values as coded from a single face may cover an interval sufficiently wide to overlap with other nominal values. Thus, the representation of a Value 1 may overlap with the nominal Value 2, and so forth. Presumably, the coded intervals might be so wide as to produce modes at unrepresented values in the distribution, if the values are points of overlap in the intervals coded for represented values.

In Experiment 1 , the void in the multidimensional space was represented by a gradual decrease in density from the extremes to a zero density in the center of the distribution. In Experiment 2, the void was made larger and abrupt, with respect to the density of the distributions, in an attempt to determine if attentional factors or a lack of discrimination among the successive values of the dimension might play a role in the emergence of a "central tendency" in the representation of the distribution of faces.

\section{EXPERIMENT 2}

\section{Method}

Subjects. Subjects were 16 undergraduate psychology students obtained in a manner identical to that in Experiment 1. They were assigned to the two experimental conditions in the order that their signatures appeared on sign-up sheets. It was assumed that the large number of random variables operating on the sign-up order were sufficient to insure random assignment.

Materials. An acquisition set of eight faces was assembled such that Values 1 and 5 on each dimension were represented four times each. Values 2, 3, and 4 were not represented. The recognition-test set was identical to that in Experiment 1 .

Procedure. Two groups were utilized, one of which did not receive instructions as to the identity of the dimensions of variability, and one of which received such instructions. The instructions consisted simply of telling the subjects that the faces would vary in three ways: The face would get longer and shorter, the nose would get longer and shorter, and the age would change. All other procedures remained identical to those in Experiment 1.

\section{Results}

A 2 (between) by 15 (within) analysis of variance was performed on the data. The value of alpha was set 


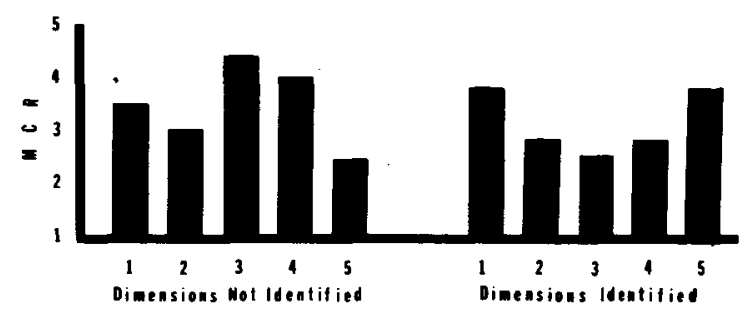

Figure 2. Mean confidence ratings for the five correlatedvalue test patterns in Experiment 2.

at .05 . The results indicate no difference in the mean confidence ratings across test stimuli due to the instruction variable $(\mathrm{MS}=0.0, \mathrm{MSe}=1.84)$. There were reliable differences among test stimuli, however $[\mathrm{MS}=3.45, \quad \mathrm{MSe}=1.84, \quad \mathrm{~F}(14,196)=1.87], \quad$ and $\mathrm{a}$ significant interaction between test stimuli and instruction condition $[\mathrm{MS}=3.36, \quad \mathrm{MSe}=1.84$, $F(14,196)=1.82]$.

The means across correlated-value test stimuli in Figure 2 serve to illustrate the nature of the Instruction by Test Stimuli interaction. With no dimensional instructions, the distribution of confidence ratings is in accordance with the predictions of the prototypedistance model, with lower mean ratings for peripheral values. With dimensional instructions, the orders are reversed, with the peripheral values receiving the higher confidence ratings, as predicted by the attributefrequency model.

Regression analyses corroborated the basic findings. Table 2 indicates that the regression coefficients are negative in the condition which incorporated no dimensional instructions, as predicted by the prototypedistance model, and positive in the condition in which dimensional instructions were incorporated, as predicted by the attribute-frequency model.

\section{Discussion}

From a general point of view, the results of Experiment 2 indicate that under some conditions the results are compatible with the prediction of a prototype-distance model and under other conditions they are compatible with the prediction of the attributefrequency model. The nature of the conditions under which each of the models successfully predicts the outcome is not at all clear, however. One interpretation is that there exists in memory a well developed schema describing the nature and range of variability of faces, with which all subjects doubtlessly have a great deal of experience. Under most circumstances, it is cognitively economical not to expend a great deal of cognitive "work" in processing a group of faces, instead making the implicit assumption that any group of faces will be congruent, within tolerable limits, to the specifications of the schema. Such a mechanism is precisely the nature of the hypotheses advanced by Oldfield (1954) as to how information reduction might occur in the coding of similar patterns. However, when attention is directed to the exact nature of local variation in the exemplars, the processing of the exemplars takes place independent of the extant schema, and the results mirror local distributions. This interpretation assumes that the general class of "Faces" varies in such a manner that the central values are, in fact, more frequent in the general population than are the peripheral values. The present author has no data supporting this notion. However, it seems, at least intuitively, that the genetic random variables might be so distributed.

Another interpretation is that, at some point of scalar separation between modes of the distribution, the multidimensional space is divided into two subspaces and two prototypes formed with Values 1 and Values 5. This interpretation might be viable if the space were unidimensional. However, the structure of the multidimensional space is such that a separate prototype would have to be formed at the intersection of Values 1 and 5 on each dimension. In the present case, that would mean that each of the acquisition exemplars would have to become a prototype, and thus there would be no means by which to predict the obtained regular differences in the recognition confidence ratings. This interprefation also fails to predict the interactive effect of instructions.

A third interpretation is wholly consistent with the attribute-frequency model. This interpretation would rely on the nature of sequential discrimination for its interpretation. Since the discrimination interval in a sequential discrimination task is much wider than in simultaneous discrimination, it might be hypothesized that, without the dimensional instructions to direct attention to the discrimination task implicitly required

Table 2

Regression Analyses for Experiment 2

\begin{tabular}{|c|c|c|c|c|c|c|}
\hline $\begin{array}{c}\text { Independent } \\
\text { Variable }\end{array}$ & $\begin{array}{l}\text { Regression } \\
\text { Coefficient } \\
\end{array}$ & $\begin{array}{c}\text { Regression } \\
\text { Mean Square } \\
\end{array}$ & $\begin{array}{c}\text { Error } \\
\text { Mean Square } \\
\end{array}$ & df & $\mathbf{F}$ & $\mathrm{p}$ \\
\hline & \multicolumn{6}{|c|}{ No Dimensional Instructions } \\
\hline \multirow[t]{2}{*}{ Frequency Sums } & $\begin{array}{l}-.195 \\
-.062\end{array}$ & $\begin{array}{l}2.2546 \\
1.4439\end{array}$ & $\begin{array}{l}.4724 \\
.5347\end{array}$ & $\begin{array}{l}1,13 \\
1.13\end{array}$ & $\begin{array}{l}4.7732 \\
2.7003\end{array}$ & $\begin{array}{l}.05 \\
.12\end{array}$ \\
\hline & \multicolumn{6}{|c|}{ Dimensional Instructions } \\
\hline $\begin{array}{l}\text { Distance } \\
\text { Frequency Sums }\end{array}$ & $\begin{array}{l}+.133 \\
+.061\end{array}$ & $\begin{array}{l}1.0471 \\
1.3840\end{array}$ & $\begin{array}{l}.1995 \\
.1735\end{array}$ & $\begin{array}{l}1,13 \\
1,13\end{array}$ & $\begin{array}{l}5.2502 \\
7.9754\end{array}$ & $\begin{array}{l}.04 \\
.02\end{array}$ \\
\hline
\end{tabular}


to analyze and store representations of similar stimuli, the intervals which are coded on the continuous dimensions of variability are very wide, overlapping the nominal experimenter-created and numbered values. If discrimination were so rough, for example, that one interval was from 1 to 3 , the next from 2 to 4 , and the last from 3 to 5 , a "central tendency" would obtain, since Value 3 would be coded when either a Value 1 or a Value 5 was experienced. The effect of dimensional instructions might be hypothesized as that of reducing the discrimination interval through attention mechanisms, thus leaving Value 3 uncoded, and producing the depression in confidence ratings in the center of the distribution.

The first interpretation will be referred to here as the local vs global schema hypothesis. It may be tested in a variety of ways. One way is through an instructional variable which does, in one condition, and does not, in the other, focus the subject's attention on the importance of creating a local schema representing the category to be instantiated in the experimental condition. Another is creating a distribution which is so at variance with the global schema that a local schema must be constructed. A third way is to compare performance on a category for which a global schema cannot exist (from lack of prior experience) to performance on a category for which a global schema is presumed to exist, under conditions in which the nature of the variance and the distributions of values are similar.

The third interpretation may be tested by including more than one level of discriminability of stimuli for which there can exist no global schema. With highly discriminable values, the distribution of confidence should be representative of the frequency distribution of the acquisition set, whereas, with less discriminable values, there should be an increment in the confidence ratings of the central-valued stimuli.

\section{EXPERIMENT 3}

\section{Method}

Subjects. Subjects were 120 undergraduate psychology students obtained and assigned in a manner identical to that of Experiment 1 .

Procedure. Three different populations of stimuli were constructed. The first population consisted of faces used in the previous experiments. The faces varied in age (which was accomplished by the number of age lines overlayed on the face), in the length of the nose, and in the overall length of the face.

The second population was a set of abstract stimuli, which nevertheless varied on the same perceptual dimensions and bore close structural resemblance to the faces. The highdiscrimination population was composed of large red rectangles, $100 \mathrm{~mm}$ in width and varying from $100 \mathrm{~mm}$ to $300 \mathrm{~mm}$ in length, in increments of $25 \mathrm{~mm}$. Each rectangle was divided in half by a black horizontal line, $100 \mathrm{~mm}$ long. Below the line and centered horizontally was a yellow rectangle, $25 \mathrm{~mm}$ in width and varying from $37.5 \mathrm{~mm}$ to $87.5 \mathrm{~mm}$ in length, in increments of $12.5 \mathrm{~mm}$. The upper edge of the rectangle was always against the horizontal line. Above the line, centered horizontally, was a pink rectangle, which was uniformly $62.5 \mathrm{~mm}$ high and $75 \mathrm{~mm}$ wide. This rectangle was divided vertically into $n+1$ equal partitions by the placement of one, two, three, four, or five black lines. The dimensions of variability were thus the length of the large red rectangle, corresponding to the length of the face, the length of the enclosed yellow rectangle, corresponding to the length of the nose, and the number of lines in the enclosed pink rectangle, corresponding to the number of age lines on the face. As with the faces, each dimension of variability incorporated five values.

The third population was identical in structure to the second with the exception that the length of the large red rectangle varied from $250 \mathrm{~mm}$ to $300 \mathrm{~mm}$ in $12.5-\mathrm{mm}$ increments, the length of the yellow enclosed rectangle varied from $62.5 \mathrm{~mm}$ to $87.5 \mathrm{~mm}$ in $6.25-\mathrm{mm}$ increments, and the number of lines in the pink rectangle varied from five to nine. The difference between high-discriminability and low-discriminability abstract patterns thus lay in the proportion of increase or decrease which separated the nominal values along the dimensions of variability.

From each population, two acquisition sets were constructed. One set, numbering eight exemplars, represented Values 1 and 5 three times, Values 2 and 4 once, and Value 3 was not represented, on all dimensions. The other acquisition set of eight exemplars represented Values 1 and 5 four times each, and Values 2, 3, and 4 were not represented. A total of six different acquisition sets (two from each of three populations) was assembled.

The recognition sets of 15 exemplars were identical to those of Experiment 2, with the exception that two additional sets, representing the same combinations of nominal values, were constructed from the two abstract populations.

The remainder of the procedure was identical to that in previous experiments, with the following exceptions. Whether the dimensions were labeled or not labeled before acquisition became a variable, as in Experiment 2, and, in the general instructions, the statement "try to remember the exact faces that you see" was expanded to include "so that later if I show you a face that you saw, you will remember having seen it, but if I show you a face that is very much like one you saw, but not identical, you will know that you didn't see it." For conditions in which the exemplars were not faces, the term "patterns" was substituted for "faces."

The design was a 3 (stimulus populations) by 2 (instruction conditions) by 2 (instantiating distributions) (between) by 15 (test exemplars) (within) factorial. Each of the 12 cells had 10 subjects assigned to it.

\section{Results}

Regression analyses were performed on each cell, using distance and frequency sums as the predictor variables. Table 3 lists the results of the 24 analyses. In general, the results indicate that no cell both regressed significantly on the prototype-distance measure and attained the appropriate negative slope. All highdiscriminability cells with nonexperienced patterns regressed significantly on frequency sums. In the lowdiscriminability cells with nonexperienced patterns, the cells which utilized only the extreme values in the acquisition set (U distribution) regressed significantly on frequency sums. All six such cells attained the appropriate (positive) slope. The other two cells, which utilized representations of less extreme values in the acquisition sets, failed to regress significantly on either measure. In the cells which utilized faces as stimuli, no significant regression coefficients were obtained on either measure. 
Table 3

Regression Analyses for Experiment 3

\begin{tabular}{|c|c|c|c|c|c|c|c|}
\hline \multicolumn{2}{|r|}{ Cell } & $\begin{array}{r}\text { Regression } \\
\text { Coefficient } \\
\end{array}$ & $\begin{array}{l}\text { Regression } \\
\text { Mean Square } \\
\end{array}$ & $\begin{array}{c}\text { Error } \\
\text { Mean Square } \\
\end{array}$ & df & $\mathbf{F}$ & $\mathbf{p}$ \\
\hline & & \multicolumn{6}{|c|}{ Distance } \\
\hline Face & $\mathrm{V}^{*} \mathrm{SI} \mathrm{I}^{*}$ & -.0241 & .0348 & .4401 & 1,13 & .0791 & $.77+\dagger$ \\
\hline Face & V DI & -.0292 & .0508 & .5891 & 1,13 & .0862 & $.77+\dagger$ \\
\hline Face & U SI & +.0675 & .2711 & .2189 & 1,13 & 1.2385 & .29 \\
\hline Face & U DI & -.0131 & .0102 & .4088 & 1,13 & .0250 & $.85 \dagger \dagger$ \\
\hline $\mathrm{HD} \dagger$ & V SI & +.4624 & 12.7442 & .2563 & 1,13 & 49.7250 & .01 \\
\hline HD & V DI & +.1594 & 1.5143 & .2661 & 1,13 & 5.6906 & .03 \\
\hline $\mathrm{HD}$ & U S I & +.3644 & 7.9154 & .5448 & 1,13 & 14.5300 & .01 \\
\hline HD & U DI & +.5091 & 15.4449 & .5747 & 1,13 & 26.8747 & .01 \\
\hline LD & V S I & +.0356 & .0754 & .2309 & 1,13 & .3266 & .58 \\
\hline LD & DI & +.0114 & .0078 & .1577 & 1,13 & .0392 & .81 \\
\hline $\mathrm{LD}$ & SI & +.1157 & .7988 & .5811 & 1,13 & 1.3746 & .26 \\
\hline \multirow[t]{2}{*}{ LD } & DI & +.4459 & 11.8540 & .6618 & 1,13 & 17.9118 & .01 \\
\hline & & \multicolumn{6}{|c|}{ Frequency Sums } \\
\hline Face & SI & -.0131 & .0264 & .4407 & 1,13 & .0600 & .80 \\
\hline Face & DI & -.0209 & .0672 & .5879 & 1,13 & .1142 & .74 \\
\hline Face & SI & +.0301 & .3542 & .2155 & 1,13 & 1.6667 & $.22+\dagger$ \\
\hline Face & DI & -.0006 & .0001 & .4095 & 1,13 & .0004 & .93 \\
\hline HD & SI & +.2803 & 12.1319 & .3034 & 1,13 & 39.9869 & $.01 \dagger \dagger$ \\
\hline HD & DI & +.0874 & 1.1804 & .2919 & 1,13 & 4.0456 & $.06 \dagger \dagger$ \\
\hline HD & SI & +.1531 & 9.1477 & .4500 & 1,13 & 20.3294 & $.01 \dagger \dagger$ \\
\hline HD & DI & +.2109 & 17.2500 & .4275 & 1,13 & 40.6003 & $.01+\dagger$ \\
\hline LD & SI & +.0159 & .0392 & .2337 & 1,13 & .1667 & $.69+\dagger$ \\
\hline $\mathrm{LD}$ & DI & +.0093 & .0134 & .1572 & 1,13 & .0854 & $.77+\dagger$ \\
\hline LD & S I & +.0768 & 2.3053 & .4652 & 1,13 & 4.9552 & $.05 \dagger \dagger$ \\
\hline LD & U DI & +.2025 & 16.0024 & .3427 & 1,13 & 46.6963 & $.01+\dagger$ \\
\hline
\end{tabular}

*V represents a frequency distribution of 31013 across the five values of each dimension, $U$ represents a frequency distribution of 40004

* $S I=$ simple (no dimensional) instructions, $D I=$ dimensional instructions

$\dagger H D=$ high discriminability, $L D=$ low discriminability

t+Regression slopes appropriate to independent variable

From the 15 test stimuli, the five correlated-value stimuli were selected for further analysis, based on the rationale that, since these comprised the first block of stimuli, they would be the most free of any interference or distortion accumulated during the test period. They also provide a neat cross-section through the distribution.

A trend analysis was performed on these stimuli within the framework of an analysis of variance in accordance with procedures detailed in Grant (1956). All hypotheses regarding differences in the distributions between cells center on the quadratic component of the distributions. As in previous analyses, the value of alpha was set at .05 . The results indicate a significant overall trend $[\mathrm{MS}=39.5767, \mathrm{MSe}=1.70$, $F(4,432)=23 \cdot 24]$. The linear component across the five correlated-value stimuli was not statistically significant $[\mathrm{MS}=2.0833, \mathrm{MSe}=1.9046, \mathrm{~F}(1,108)=1.09]$. The quadratic component was statistically significant $[\mathrm{MS}=134.87, \quad \mathrm{MSe}=2.1184, \quad \mathrm{~F}(1,108)=63.67]$; the cubic component was statistically significant $[\mathrm{MS}=21.33, \quad \mathrm{MSe}=1.4769, \quad \mathrm{~F}(1,108)=14.45]$; and the quartic component was not significant $(\mathrm{MS}=.0233, \mathrm{MSe}=1.3112)$.

Of the interactions between polynomial components and groups, only the Groups by Quadratic
$[\mathrm{MS}=13.5641, \mathrm{MSe}=2.1184, \mathrm{~F}(11,108)=6.403]$ and the Groups by Cubic [MS $=4.2096, \quad \mathrm{MSe}=1.3112$, $\mathrm{F}(11,108)=3.21]$ were statistically significant. On the advice of Myers (1972), an attempt was made only to interpret those significant results which relate to the theoretical predictions. The overall trend indicates, predictably, that the variations across the five stimuli are orderly. Both prototype-distance and attribute-frequency theories predict that the five stimuli will be ordered, with respect to the dependent variable. Similarly, a significant quadratic component does not, by itself, differentiate between theories. However, the prototype-distance model predicts a negative weighted sum, given the order in which the orthogonal weights for this component are assigned across the dependent variable $(+2,-1,-2,-1,+2)$, whereas the attribute-frequency model predicts a positive sum. Only the cells for which the intermediate values were represented during acquisition ( $\mathrm{V}$ distribution) attained a negative sum of weighted scores. Inspection of Figures 3,4 , and 5 verifies that the general shape of the distribution in cells for which only the extreme values were shown during acquisition (U distribution) is uniformly depressed in the central region. For the $V$-distribution cells, however, inversions occur with face stimuli, yielding a slightly negative weighted sum 

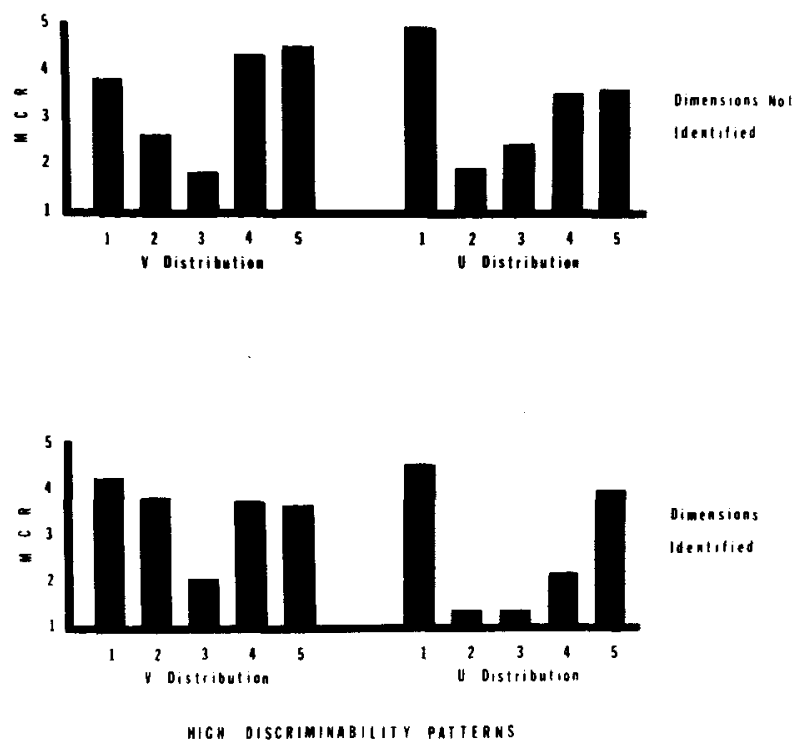

Figure 3. Mean confidence ratings for the five correlatedvalue test patterns in cells which utilized high-discriminability abstract patterns in Experiment 3.
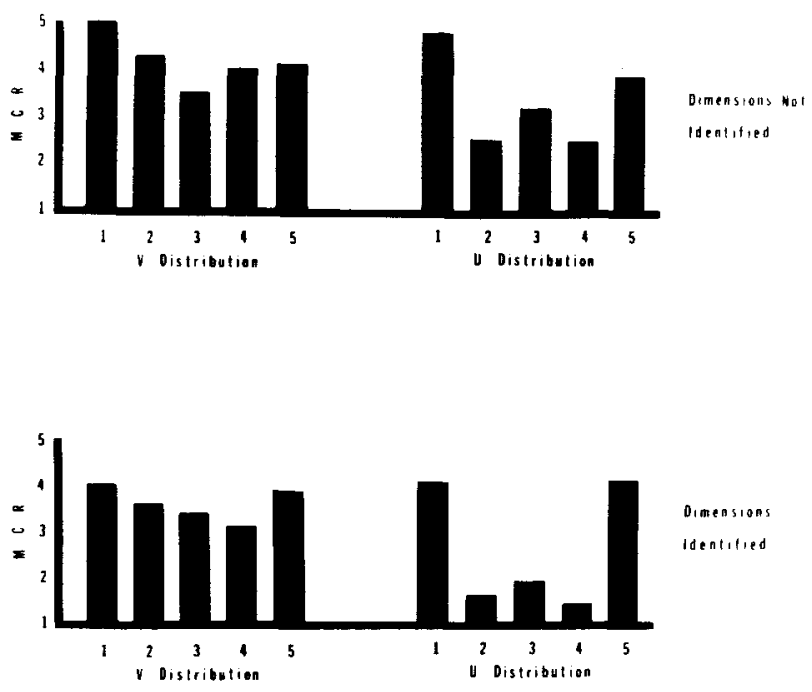

LOW OISCRIMIMABILITY PAHERHS

Figure 4. Mean confidence ratings for the five correlatedvalue test patterns in cells which utilized low-discriminability abstract patterns in Experiment 3.

when collapsed across all V-distribution cells. The significant Quadratic by Groups interaction indicates that the degree of depression at the center of the distributions changes as a function of groups. Once again, this particular result can be predicted by both theories, as a result of different frequency distributions, different instructions, different stimuli in acquisition, or combinations of those variables. To test specific hypotheses, multiple comparisons were performed in accordance with procedures detailed in Myers (1972). The attribute-frequency model with the interval-storage hypothesis predicts that faces with a $\mathrm{V}$ distribution and abstract low-discriminability patterns which are $\mathrm{V}$ distributed should differ in the quadratic component when compared to all other cells. With alpha set at .05 , this result obtained $[\mathrm{MS}=40.18, \mathrm{MSe}=2.12$, $\mathrm{F}(1,108)=18.92]$. The attribute-frequency model also predicts that, within face stimuli, the two V-distributed cells should differ from the two U-distributed cells. This result also obtained [MS $=16.90, \mathrm{MSe}=2.12$, $\mathrm{F}(1,108)=7.97]$. Exactly the same hypothesis is made about the abstract low-discriminability patterns. This result failed to obtain $[\mathrm{MS}=4.628, \mathrm{MSe}=2.12$, $F(1,108)=2.18]$. The two low-discriminability $U$ distributed cells do, however, differ significantly from the low-discriminability V-distributed cell which incorporates dimensional instructions [MS $=13.67$, $\mathrm{MSe}=2.12, \mathrm{~F}(1,108)=6.45]$.

\section{Discussion}

The first hypothesis to be considered stated that, when there exists a well developed schema describing the nature and variation of a set of stimuli, the cognitive system will simply map local variation onto the global schema and the distribution of confidence ratings will mirror the distributions of the global variations. However, if attention is directed to the local variation via instructions, or if a global schema does not exist, the distributions of confidence ratings will mirror the local distributions of similarity. This hypothesis predicts that all of the nonexperienced patterns, which were labeled "abstract," and the face
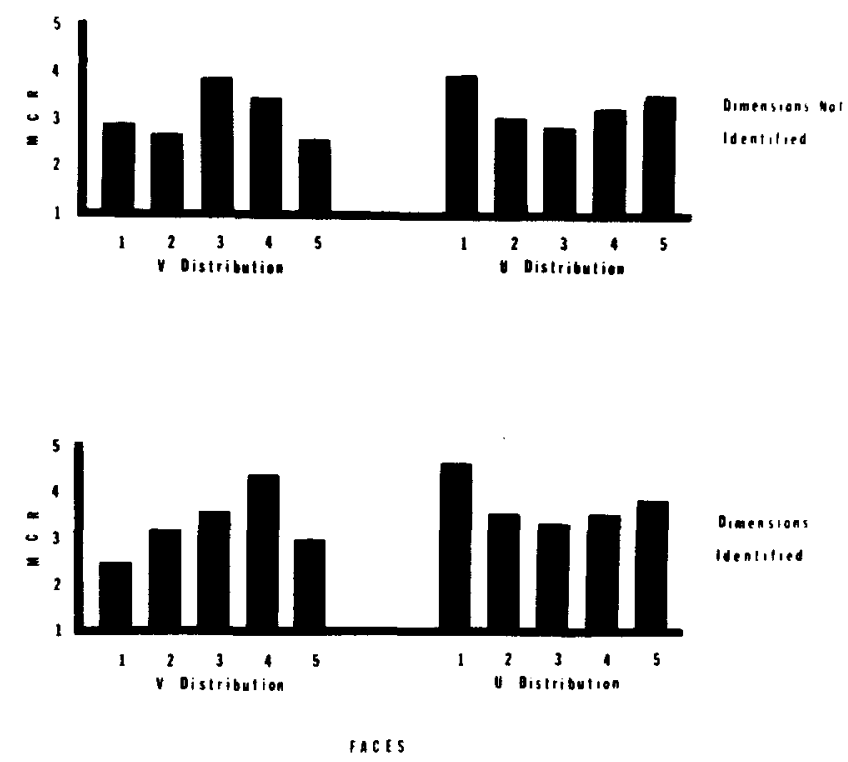

Figure 5. Mean confidence ratings for the five correlatedvalue test patterns in cells which utilized face patterns in Experiment 3. 
stimuli associated with dimensional instructions will produce recognition confidence distributions which will map onto the distributions of values in the acquisition sets. Figures 3,4 , and 5 illustrate that the partitioning of conditions by this hypothesis did not obtain. All abstract patterns produced recognition confidence rating distributions which were generally congruent with the local distributions of attributes, but the hypothesis partitions face stimuli by instruction variable, whereas the empirical partitioning is by value distribution in the acquisition set ( $\mathrm{V} / \mathrm{U}$ distribution). With faces, the $\mathrm{V}$ distribution produced the central tendency characteristic of the predictions of the prototype-distance model, whereas the $U$ distribution produced a depressed central region. The rejection of this hypothesis is by no means on indisputable grounds, however. One might hypothesize a high-tolerance monitor of local variation which accepts as within limits certain distributions (V) while rejecting others (U) as too much at variance with the global schema, activating the formation of a local schema instead. The present findings are, however, congruent with those of Rosch (1973), who found that subjects had more difficulty learning categories when the "natural" centers of categories were placed at the periphery of the experimental category, indicating that her subjects, like those in the present research, could not form a "local" schema in violation of the "global" or "natural" schema.

Another hypothesis is that there are two mechanisms for constructing schemata. When dimensionally analyzed but structurally intact representations are output by the perceptual system, the representations form intersections in a multidimensional spatial distribution, and this categorical structure is described by the prototypedistance model. However, when the output of the perceptual system is in terms of unit representation of the values of the exemplars on the various dimensions of variability, the schema is constructed in such a fashion as to represent the local distributions of values along these dimensions of variability, as predicted by the attribute-frequency model. In the making of these predictions, it has been presupposed that a representation of a face is more likely to be in holistic fashion, structurally intact, than a pattern structured from various colors and sizes of rectangles and different numbers of lines. The difference might be characterized by describing one as "an old man with a long face and short nose" and the other as "long red rectangle, medium-yellow enclosed rectangle, four lines." Note that the distinction here is not strictly one of integrable vs separable dimensions of variability (Garner, 1974); in both cases they are separable. However, in the first case, the information is output as a three-value intersection, whereas, in the second case, it is output as three discrete items of information with no cooccurrence information.

The hypothesis, then, predicts that the partitioning should be between faces and abstract patterns. The regression analyses, the multiple comparisons, and simple inspection of Figures 3, 4, and 5 indicate that the partitioning is not in this fashion. The results would seem to be on relatively firm ground, since there seems to be no viable reason why some faces would be output in a structurally intact fashion and others unidimensionally, depending on the distribution of instantiating values. Had the partitioning of faces been on the basis of instructional conditions, the rejection of this hypothesis would have been with a great deal less confidence, since bringing the subject's attention to the dimensional structure might well be associated with unidimensional output of the values from the perceptual system. Instead, partitioning is between acquisition distributions, with the $\mathrm{V}$ distribution resulting in the central tendency and the $U$ distribution resulting in the depressed central region.

The final hypothesis is the "interval-storage" hypothesis, which assumes that a value on some dimension of variability is not a point on a continuous distribution, but is, instead, an interval of some magnitude. When the intervals which represent adjacent nominal values along the dimensions of variability overlap, a central tendency is exhibited, since the frequency with which the central values are represented is incremented not only when central values are experienced, but to some degree when intermediate and peripheral values are experienced as well, depending upon the amount of overlap. However, when the size of the interval which represents each value is reduced in some manner, or when the values themselves are sufficiently separated along the dimension of variability, this effect will not obtain and no central tendency will be exhibited.

Note that this hypothesis is in no manner a reformulation of a distance hypothesis. It clearly predicts that the central tendency is a result of the overlap of the intervals which represent each nominal point on some scale, rather than the distances between points. Given a constant scalar distance between represented points, any variable which serves to increase discrimination among values would hypothetically affect the shape of the distribution of confidence ratings. This prediction cannot be derived from within the context of the distance model. Although the distance model may predict a greater range of similarity ratings due to increased discriminability (i.e., more highly discriminable stimuli may be evaluated as less similar, and thus the range of distances may be greater), there is no mechanism by which to predict a decrease in the central tendency as a result. If the effect is asymmetrical, the distance model would predict a shift in the central tendency, but, elevation of the confidence ratings at the new multidimensional center is predicted, rather than a depression, as predicted by the attributefrequency model.

This hypothesis, therefore, predicts a two-dimensional interactive partitioning of cells. It was predicted that 
the results within faces would partition by instantiating distribution, the $V$ distribution exhibiting a central tendency and the $U$ distribution exhibiting less central tendency or a central depression. These results obtained, when the first block of correlated-value stimuli are referenced. The results illustrated in Figure 5 show a central tendency effect for the V-distribution conditions and a central depression for the U-distribution conditions. This result is corroborated by the trend analysis.

The second prediction is that none of the highly discriminable abstract patterns will exhibit a central tendency. The reasoning here is that, if the values of the dimensions are highly discriminable, there will be no overlap among the intervals which represent the nominal values, and the distribution of confidence ratings will map onto the frequency distribution of values in the instantiating distributions. Although the distribution of confidence ratings among the stimuli are not as uniform as might be wished, none represents a central tendency with respect to the highly discriminable patterns (see Figure 3). Three of the four cells regressed significantly across all test patterns on the frequency sums (Table 3); the fourth cell fell just short of the level of alpha $(p<.06)$.

The third prediction depended upon choosing a fortuitous level of "low-discriminability." The prediction is that in low-discriminability abstract patterns, there should be an effect of instantiating distribution which partitions the cells in the same fashion as the ones for which faces were the stimuli. The U-distribution cells should exhibit a depressed central region and the V-distribution cells should exhibit a central tendency. The result is illustrated in Figure 4. The V-distribution cells clearly failed to exhibit a central tendency. The trend analysis failed to yield evidence of a significant difference in the quadratic components of the $\mathrm{V}$ and $\mathrm{U}$ distributions. However, a comparison between the U-distributed cell with dimensional instructions and the two $V$-distributed cells was significant. The results might be characterized as evidence of less central depression in the V-distributed cells as opposed to obtaining a central elevation.

The present author thus, in Experiment 3, finds evidence that the attribute-frequency model is capable of successfully generating predictions as to the distribution of recognition confidence. The predictions, while by no means perfect, were generally supported. The model is thus capable of accounting for results previously thought to be the exclusive domain of the prototype models. In the present case, the results predicted and obtained are widely at variance with the predictions of the prototype-distance model.

\section{GENERAL DISCUSSION}

Ultimately, this paper addresses itself to a single basic issue: Given a set of similar stimuli which vary along continuous dimensions, can a theory which hypothesizes that the internal structure of a category is represented by the frequency distributions of the experienced values along the dimensions of variability account for performance phenomena previously predictable only from within the framework of a theory which hypothesizes that a category is represented as a series of multivalued intersections in multidimensional space?

Central to the issue is the question of how a value along a continuous dimension of variability might be represented in memory. On a truly continuous distribution, the notion that a particular value can have a frequency is untenable. It has been asserted, however, that the notion that a particular point value along a continuous dimension of variability can be represer,ted is equally untenable, and that the representation in memory must be one of an interval, not a point. Representing the frequency of an interval presents no particular problem.

The next problem is that of accounting for the performance phenomenon known as the "central tendency." This phenomenon is manifested by a tendency for responses to be stronger, or more positive in some sense, to stimuli lying in the middle of the range. Its manifestation in several experimental paradigms was noted by Woodworth (1938). Within the context of the study of categories, it has generally been regarded as an index of the "membership value" of particular exemplars. Those exemplars which are best recognized or for which subjects report higher confidence in recognition or classification are assumed to be the best examples of the category.

The prototype-distance model of Posner (e.g., 1969) hypothesizes that a prototype, or best example, is derived in the center of the multidimensional distribution of experienced exemplars by the application of a sophisticated averaging technique. Specifically, the prototype is that multivalued intersection in the multidimensional space from which the total of the distances to all experienced exemplars is least. The closer in distance, and, thus, the more similar, an exemplar is to this prototype, the better member of the category it is.

The attribute-frequency model accords no special status to the best example of the category. Each exemplar is evaluated in terms of the sum of the frequencies with which its attributes occur in the category. That exemplar which is represented by the highest sum of frequencies is the best example.

The nature of the prototype-distance model is such that it predicts circumstances under which the prototype might be composed of values on the dimensions of variability which have never been experienced, should those values be the central values. The attributefrequency model would derive a sum of zero for such a pattern, predicting that it would be a very poor member of the category.

In Experiment 1, the results were unequivocal. The 
best recognized exemplar was that which incorporated the central Value 3 on each dimension of variability, despite the fact that no Value 3 had ever been experienced during acquisition.

Experiment 2 tested the central tendency against a larger unrepresented interval, and tested whether calling the subjects' attention to the local variation among faces might eliminate the central tendency. When no dimensional instructions were given, the distribution of confidence ratings exhibited the central tendency. When dimensional instructions were incorporated, the central tendency disappeared and the confidence ratings regressed significantly on the frequency sums. The finding that a relatively simple instruction manipulation can significantly alter the shape of the distribution of recognition confidence ratings is important. It indicates that the distance model, at least in present form, cannot account for the central tendency, since the model cannot predict when the tendency will and will not obtain. It is also evidence mediating against the postulation of a completely passive model of such processing. Any model must account for the manner in which the instruction manipulation alters the nature of the underlying categorical structure.

One interpretation is the local vs global schema hypothesis, which would assume that, for categories with which the subject is experienced, there exists a well defined schema, and that this serves as the reference for recognition. However, if attention is directed to immediate experience, a local schema might be constructed which more accurately represents the local similarity structure. In such a case, recognition of faces might exhibit a central tendency when the global schema is the reference, but not when the local schema is the reference.

An alternative is the interval-storage hypothesis, which assumes that the representation of values on continuous dimensions is in the form of intervals rather than points. When discrimination among values is poor, the intervals which represent these values are wide and may overlap other values. Thus, the frequencies with which these values are "experienced" may increment along with the values actually experienced, resulting in a central tendency. When discrimination among values is good, the intervals which represent these values are narrow, and the resulting frequency distribution accurately mirrors the experienced distribution.

The two hypotheses generated different predictions as to the partitioning of the cells in Experiment 3 between those which should show a central tendency and those which should not. The results support the interval-storage hypothesis.

It is, therefore, concluded that an attribute-frequency model is not limited to accounting for results of prototype-formation experiments in which the dimensions of variability vary discretely. Nor would it appear inferior to the prototype-distance model when predicting the outcome of prototype-formation experiments. From a simplified point of view, the difference between the two theoretical approaches is not one of central tendency vs no central tendency, but, instead, one of appropriate measures. Only further experimentation will determine whether it is a complex mean or a complex mode.

It is noteworthy that the research in this paper was addressed to only one of two basic problems in research on abstraction. A complete theory of the abstraction process must model the principles and processes underlying both the formation of, and the differentiation between, conceptual and perceptual categories. The present research is concerned only with the processes by which a category is formed, and not with the processes underlying classification decisions. The attribute-frequency model represents a unifying construct in the issue of whether "prototypes" or "features" are learned. The model predicts that the best example or examples are those with the highest attribute-frequency sums. The present research, as well as that of Neumann (1974), Posnansky and Neumann (1976), and Rosch and Mervis (1975) support this prediction. The prototype would, therefore, not appear to be a memory structure apart from information about exemplars. In fact, within the context of the attributefrequency model, the concept of a "prototype" has no useful attributes. Some exemplars are simply better members of the category than others, by virtue of their higher attribute-frequency sums. Thus, two or more exemplars may be "equally prototypical" of a given category.

This is not to suggest that the attribute-frequency model cannot address itself to the issue of classification across categories. To the contrary, Rosch and Mervis (1975) have presented evidence that the extension of such a model to a classification paradigm can be accomplished simply and successfully. The process of assigning membership within a category and between categories also unifies the issue of "commonality" vs "distinctiveness." Membership value within a category increases as the commonality of the exemplar's attributes and the attributes of the category increases. Assignment across categories would proceed on the same basis. An exemplar would be assigned to the category, the experienced exemplars of which incorporate the exemplar's attributes most frequently. But errors in classification would be a function of the slope of the values of the attribute-frequency sums across categories. Steeper slopes (i.e., greater differences in attribute-frequency sums) would be characterized as obtaining fewer errors in classification, which means that fewer errors are obtained when there are attributes which are distinctive of a particular category. Thus, the classification proceeds on the basis of which category has the larger number of attributes in common with the exemplar, but the error in classification is 
least when the "attributes-in-common" are distinctively distributed across categories. This model would seem to be consistent with results recently reported by Barresi, Robbins, and Shain (1975), who concluded that classification proceeds on the basis of distinctive features.

Quite obviously, the leap from the above generalizations to a logical process model cannot be accomplished without difficulty. The reader will have ascertained that the present author is attempting to account for the structure of categories and for the classification process in terms of a single underlying cognitive process which operates on a data base consisting of the output of the perceptual system, which is assumed by the model to perform feature analysis. The nature of the data base is assumed to vary with task demands and context. The portion of the data base processed is also assumed to vary. Whether such a model is viable remains to be determined. It would appear, however, to be a parsimonious alternative to the complex assumptions of other models.

\section{REFERENCES}

Barresi, J., Robbins, P.. \& Shain, K. Role of distinctive features in the abstraction of related concepts. Joumal of Experimental Psychology: Human Learning and Memory, $1975,104,360-368$.
GARNER, W. R. The processing of information and structure. New York: Lawrence Erlbaum, 1974.

Grant. D. Analysis-of-variance tests in the analysis and comparison of curves. Psychological Bulletin, 1956, 53. $141-154$.

MYers, J. L. Fundamentals of experimental design. Boston: Allyn and Bacon, 1972.

Neumann, P. G. An attribute frequency model for the abstraction of prototypes. Memory \& Cognition, 1974, 2, 241-248.

Oldfield, R. C. Memory mechanisms and the theory of schemata. British Journal of Psychology, 1954, 45, 14-23.

Posnansky, C. J., \& Neumann, P. G. The abstraction of visual prototypes by children. Journal of Experimental Child Psychology, 1976, 21, 367-379.

PosNer, M. I. Abstraction and the process of recognition. In G. H. Bower \& J. T. Spence (Eds.), The psychology of learning and motivation (Vol. 3). New York: Academic Press, 1969.

Rosch, E. On the internal structure of perceptual and semantic categories. In T. M. Moore (Ed.), Cognitive development and the acquisition of language. New York: Academic Press, 1973.

Rosch, E., \& MERvis, C. Family resemblances: Studies in the internal structure of categories. Cognitive Psychology, 1975, 7, 573-605.

Woodworth, R. S. Experimental psychology. New York: Henry Holt, 1938.

(Received for publication June 24, 1976; revision received September 21, 1976.) 